\title{
Preliminary Meteorological Results of a Four-Dimensional Data Assimilation Technique in Southern Italy
}

\author{
Elenio Avolio $^{{ }^{*}}$, S. Federico ${ }^{1}$, A. M. Sempreviva ${ }^{1}$, C. R. Calidonna ${ }^{1}$, L. De Leo ${ }^{1}$, C. Bellecci ${ }^{2,3}$ \\ ${ }^{1}$ ISAC-CNR, Lamezia Terme, Italy \\ ${ }^{2}$ CRATI Scrl, c/o University of Calabria, Rende, Italy \\ ${ }^{3}$ University of Rome “Tor Vergata", Department STFE, Rome, Italy \\ E-mail:e.avolio@isac.cnr.it \\ Received April 27, 2011; revised June 3, 2011; accepted June 18, 2011
}

\begin{abstract}
A four-dimensional data assimilation (FDDA) scheme based on a Newtonian relaxation (or "nudging") was tested using observational asynoptic data collected at a coastal site in the Central Mediterranean peninsula of Calabria, southern Italy. The study is referred to an experimental campaign carried out in summer 2008. For this period a wind profiler, a sodar and two surface meteorological stations were considered. The collected measurements were used for the FDDA scheme, and the technique was incorporated into a tailored version of the Regional Atmospheric Modeling System (RAMS). All instruments are installed and operated routinely at the experimental field of the CRATI-ISAC/CNR located at $600 \mathrm{~m}$ from the Tyrrhenian coastline. Several simulations were performed, and the results show that the assimilation of wind and/or temperature data, both throughout the simulation time (continuous FDDA) and for a $12 \mathrm{~h}$ time window (forecasting configuration), produces improvements of the model performance. Considering a whole single day, improvements are substantial in the case of continuous FDDA while they are smaller in the case of forecasting configuration. Enhancements, during the first six hours of each run, are generally higher. The resulting meteorological fields are finalised as input into air quality and agro-meteorological models, for short-term predictions of renewable energy production forecast, and for atmospheric model initialization.
\end{abstract}

Keywords: Data Assimilation, Short Term Forecast, Mesoscale Model Performance

\section{Introduction}

A particularly crucial issue for improving mesoscale numerical models is the improvement of knowledge of the atmospheric state through the use of available data, in order to produce good initial and boundary conditions. Several instruments, such as profilers, sodars, radars, satellite systems and surface meteorological stations, are able to provide continuous streams of data on evolving atmospheric conditions, and also provide spatial information in vertical or horizontal space.

Data assimilation is the procedure that consists to incorporate observational data into analysis or forecast provided by meteorological model [1].

Four Dimensional Data Assimilation (FDDA), based on Newtonian Relaxation (or nudging) [2,3], is a useful and relatively simple data assimilation technique to produce accurate meteorological simulations. Nudging adds an extra tendency term to the prognostic equation of the assimilated variable, and forces the predicted variable towards the available observations. Since nudging is performed toward observations, the technique is referred to as "observational data assimilation (ODA)"

Several studies, referred to the regional and local scales, have shown that FDDA, on average, can reduce errors by about $25-60 \%$, depending on the cases [4-7]. In this sense, the most common problem is to obtain an independent data set for effective validation of diagnostic models [8].

Recent studies describe experiments were verification data differ from those used for assimilation. In Tanrikulu et al. [9] and in Michelson and Seaman [10] is used the data withholding technique. Also Nielsen-Gammon et al. [11], more recently, use this approach of validation. Umeda and Martien [12] use different data set for verification, and Barna and Lamb [13] perform an indirect verification, analyzing the final results of air quality simulations. 
In this work, we present results from a preliminary direct verification approach carried out assimilating atmospheric parameters, both at the surface and above, at a specific site ("LAM" experimental site). In particular, we assimilated vertical wind profiles from the sodar and wind profiler, and wind, temperature and specific humidity from the surface meteorological station. A tailored version of model RAMS was run at high spatial horizontal resolution $(1 \mathrm{~km})$, to investigate the improvements of the model performance obtained by the assimilation. The model verification consists in comparing the simulated parameters (in particular the surface temperature) both with those measured in LAM station (direct and dependent verification) and with those measured at a second experimental site ("SUF" station), locate few kilometres to the NE of the LAM (direct and independent verification). The RAMS meteorological fields, simulated with and without data assimilation, were evaluated and compared for selected case studies in a 10 days study-period, from 8 to 17 August 2008. Several experiments were performed for each case (assimilation for the entire simulation time, and for different time windows).

\section{Experimental Setup}

\subsection{Study Area}

Our analyses were conducted in the Calabria region, the southernmost tip of the Italian peninsula located in the Central Mediterranean Basin (Figure 1(a)). The region extension (Figure 1(b)) ranges between $38^{\circ} 12^{\prime}$ and $40^{\circ}$ latitude North and between $16^{\circ} 30^{\prime}$ and $17^{\circ} 15^{\prime}$ longitude East. The west coast of Calabria is bounded by the Tyrrhenian Sea, while the East and South coasts by the Ionian Sea. The Apennines Mountains chain runs north to south along the coast, and the region is characterized by five main topographical features reaching 1500 - 2000 m elevations: Pollino, Catena Costiera, Sila, Serre, Aspromonte. The average width of the region is about 50 $\mathrm{km}$ in the west-east direction and $300 \mathrm{~km}$ in north-south direction. There are three main planes by the sea (Sibari, Gioa Tauro, Lamezia) where most of agricultural and industrial areas are placed. The experimental field used for our analysis is located in the Lamezia Plane.

\subsection{Surface Stations}

We considered observations from two surface stations; the first one is located in the experimental site and is considered both for the verification and for the assimilation ("LAM" station). The second station is located few kilometres to the NE of the experimental field and provides independent data for verification ("SUF" station). The LAM surface station measures temperature, pres-

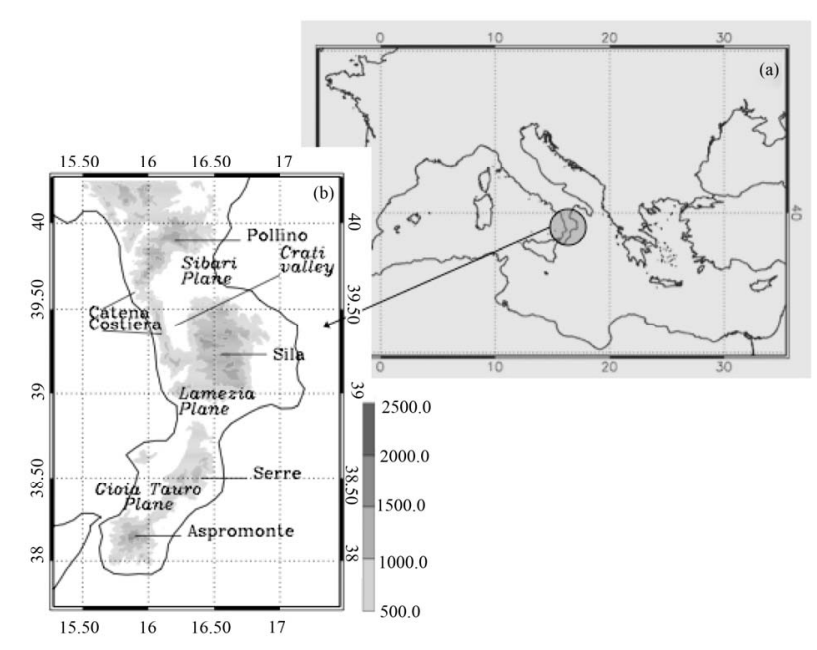

Figure 1. (a) Calabria region in the central Mediterranean; (b) Calabria features cited into the text. Grey shading shows the topographic height (m).

sure, global solar radiation, wind speed and direction (10 $\mathrm{m}$ above ground level), precipitation, relative humidity, and soil temperature at $10 \mathrm{~cm}$ depth. The SUF station measures only temperature, precipitation, and relative humidity. The parameters considered for the assimilations are: temperature $T$, relative humidity $R H$, and wind $W$. Relative humidity, before being assimilated into the model is converted to specific humidity. For the verification we consider temperature only, both for LAM and SUF station. Data are available and assimilated every 15 minutes.

\subsection{Radar Wind Profiler}

A radar wind profiler operated at the experimental site in summer $2008[14,15]$. The instrument sounds the lower troposphere, usually up to $3 \mathrm{~km}$, and measures the horizontal and vertical wind components using one vertical and two oblique beams slanted at an off-zenith angle of $15.5^{\circ}$. The operating frequency is $1290 \mathrm{MHz}$ (about $23 \mathrm{~cm}$ wavelength). Returned echoes are due to air masses refractive index fluctuations generated by the wind. Data consist of the three wind components (zonal, meridional and vertical). The vertical resolution is $100 \mathrm{~m}$, the minimum range gate is $150 \mathrm{~m}$, and the vertical range is 2 to 5 $\mathrm{km}$ depending on atmospheric conditions. Accuracy of the wind measurements is less than $1 \mathrm{~m} / \mathrm{s}$ for the horizontal wind components, $0.5 \mathrm{~m} / \mathrm{s}$ for the vertical component, and less than $10^{\circ}$ for the direction. Data are available every 30 minutes.

\subsection{DOPPLER Sodar}

Another instrument taken into account is a Doppler SO- 
DAR, which operates at the experimental site [14]. The SODAR transmits short acoustic pulses of a certain frequency into the atmosphere. A small fraction of the acoustic energy is scattered back from density fluctuations in the atmosphere. Because these micro-turbulent fluctuations move with the mean wind flow, the frequency of the backscattered signal is shifted according to the wind component parallel to the propagation of the acoustic waves (Doppler shift). The SODAR used in this work emits short pulses at $1900 \mathrm{~Hz}$, using a phased array with 24 loudspeakers. Measurements consist of vertical profiles of wind speed components (horizontal and vertical) and turbulence with a vertical resolution of $10 \mathrm{~m}$. The maximum vertical range is $300 \mathrm{~m}$; however, the effective vertical range depends significantly on the ambient noise. The lowest range gate is $35 \mathrm{~m}$. Data are available every 10 minutes.

\section{Model Configuration and Simulations}

\subsection{RAMS Description}

We used the meteorological model RAMS in its version 6.0, daily operational at ISAC-CNR (http://meteo.crati.it/ previsioni.html) and already adopted in several previous works $[16,17]$. A detailed description of the RAMS model is given in Cotton et al. [18].

The model is configured with four two-way nested grids (Figure 2). Horizontal resolutions are $27 \mathrm{~km}, 9 \mathrm{~km}$, $3 \mathrm{~km}$ and $1 \mathrm{~km}$ respectively. The fourth grid is centred over Lamezia Terme experimental site. Thirty vertical levels are used, from the surface up to $16,300 \mathrm{~m}$ of altitude in the vertical coordinates that follow the terrain. Levels are not equally spaced: layers within the Planetary Boundary Layer (PBL) are between 23 and $200 \mathrm{~m}$ thick, whereas layers in the middle and upper troposphere are $1000 \mathrm{~m}$ thick. Precipitation is calculated both by an explicit prognostic method for seven hydrometeors (rain, cloud droplets, hail, graupel, pristine, snow and aggregates) and with a convective scheme that is activated for the first and the second grid. The explicit RAMS microphysics scheme is shown in Walko et al. [19]. Convective precipitation is parameterized following Molinari and Corsetti [20] who proposed a simplified form of the Kuo scheme, which includes the downdraft effect. The parameterization of the surface layer and the energy/momentum transfer between the atmosphere, the biosphere and the soil, follow the LEAF-3 scheme [21]. Radiation is parameterized according to the Chen-Cotton scheme, which includes the effect of clouds [18]. Turbulent processes are calculated according to the scheme of Mellor-Yamada [18,22], which employs a prognostic turbulent kinetic energy.

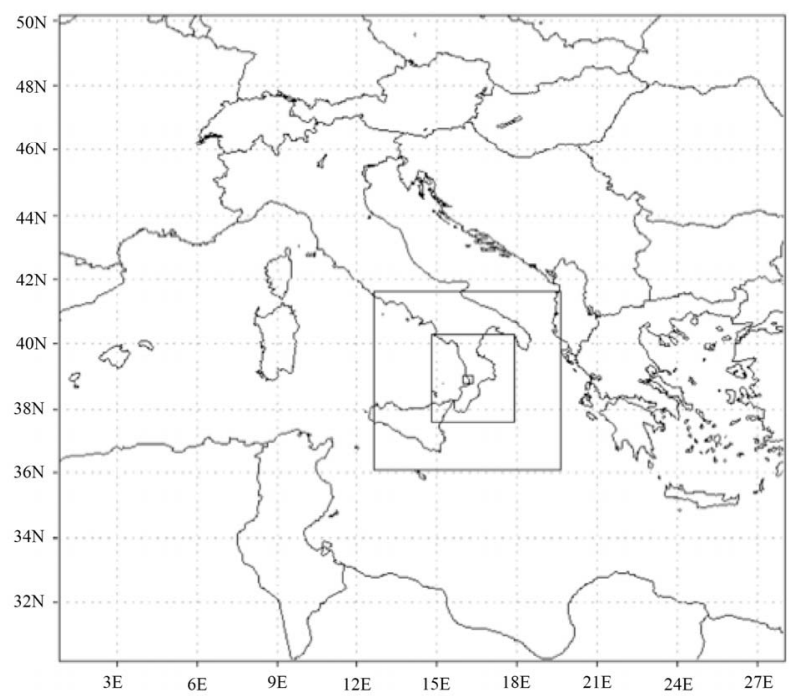

Figure 2. RAMS model domains. Horizontal grid resolutions are $27 \mathrm{~km}$ for the domain $1,9 \mathrm{~km}$ for the domain 2,3 $\mathbf{k m}$ for the domain 3 and $1 \mathrm{~km}$ for the domain 4 .

Atmospheric initial and dynamic boundary conditions are derived from ECMWF forecast for the simulated day. The RAMS model output is stored hourly, and each run lasts 36 hours. We have considered 10 days, from 8 to 17 August 2008, and each day is simulated with an individual RAMS execution. Each run starting at 12 UTC of the previous day and the first $12 \mathrm{~h}$ are considered for model spin-up.

\subsection{Algorithm of 4DDA (Nudging)}

For the assimilation procedure, a Newtonian relaxation (or nudging) was adopted [2,3]. The method consists of adding a term to the prognostic equations that nudges the predicted variables toward available observations (interpolated in each model grid). The nudging term is given by:

$$
\frac{\partial \phi_{m}}{\partial t}=\frac{\left(\phi_{o b s}-\phi_{m}\right)}{\tau}
$$

$\phi_{m}$ is the prognostic model variable, $\phi_{o b s}$ is the measured variable, and $\tau$ is a relaxation time scale. The relaxation time scale is chosen following empirical considerations. If $\tau$ is very small, the solution converges toward the observations too fast, and the dynamics do not have enough time to adjust; conversely, if $\tau$ is too large the errors of the model can develop too much and the nudging does not have enough time to become effective. Several studies have demonstrated that $\tau$ should be chosen so that the last term is similar in magnitude to the less dominant terms. Typical values of $\tau$ are between 15 minutes (strong) and 3 hours (weak). Hoke and An- 
thes [23], for example, used a very short time scale (20 minutes) in their experiments, while Stauffer and Seaman [4] adopted about 1 hour in their work.

The spatial variation of nudging is:

$$
f(r)=e^{-r / r_{0}}
$$

$r$ is the distance from the measuring point, and $r_{0}$ is a reference distance (or radius of nudging) that represents the range of the nudging; the value $r=0$ corresponds to the position of Lamezia Terme experimental site. The weight of the nudging is obtained by multiplying the term (1) and (2). Clearly, the choice of $r_{0}$ and $\tau$ will play a key role. After several preliminary tests we have chosen, for our experiments, a relaxation time scale of $900 \mathrm{sec}$. (strong nudging) and a radius of nudging of $10 \mathrm{~km}$ (meso- $\gamma$ scale).

Since the acquisition times and the spatial ranges of the three instruments are different (Section 2). In order to obtain a unique time of assimilation, we combined the assimilated data as follow:

Surface station data (T, RH and $\mathrm{W}$ ) are assimilated every 15 minutes. At upper levels, the vertical profile of $\mathrm{W}$ is obtained by combining sodar and wind profiler data between $35 \mathrm{~m}$ and $200 \mathrm{~m}$; above $200 \mathrm{~m}$, instead, is considered only the wind profiler. First, wind profiler and sodar measurements are averaged over 30 minutes; subsequently, all the data are combined in order to build a unique measured profile every 3 hours $(00,03,06, \ldots, 21$ UTC), which is nudged into the model.

\subsection{Simulations Description and Statistics}

For each day, we have performed three numerical experiments for several different cases. In particular, we considered one simulation without assimilation (T00 W00, corresponding to reference simulation), one simulation assimilating data for the entire simulation time (T36_W36, corresponding to continuous FDDA), and one simulation assimilating data for a specific time window (T12_W12, corresponding to forecasting configuration). It is useful to remember that each run lasts 36 hours, and the first $12 \mathrm{~h}$ are considered for model spinup.

MAE (Mean Absolute Error) and RMSE (Root Mean Square Error) are used for statistics.

$$
\begin{aligned}
\text { MAE } & =\frac{\sum_{i=1}^{N}\left|x_{i}-x_{o i}\right|}{N} \\
R M S E & =\sqrt{\frac{\sum_{i=1}^{N}\left(x_{i}-x_{o i}\right)^{2}}{N}}
\end{aligned}
$$

$x_{i}$ are the simulated data, $x_{o i}$ are the observations, and $N$ is the number of data.

MAE and RMSE are computed for T (surface temperature) and for both surface stations (LAM and SUF). We calculated the daily MAE and RMSE to estimate the complete simulation performances, and the 6h-window MAE and RMSE to estimate the short-term forecast performances. In particular, for each day we computed the error statistics between 00 UTC and 06 UTC for the short-term, and between 00 UTC and 23 UTC for the complete forecast. Obviously, T00_W00 is the reference simulation type and all experiments are referred to it.

\section{Results}

Table 1 summarizes all the obtained results. To help reading, we remark that:

- The upper half of the table refers to temperature MAE, while the lower half refers to temperature RMSE;

- MAE and RMSE are reported both for LAM and for SUF;

- For each station the error is shown for the complete run and for the first 6 hours;

- Both for complete simulation and for the short term forecast performances are reported errors related to T00 _W00, T36_W36 and T12_W12 experiments;

- Ten days are considered, from 8 to 17 August 2008. Table 1 shows the error for each day, as well as their average;

- For brevity, we focus our attention on the RMSE only; similar conclusions can be reached by considering the MAE. In order to catch a better confidence with the obtained results, we calculated and show the percentage of improvement for each run type (the calculation is performed respect to the T00_W00 simulation);

- Most significant results are highlighted with letters (a, b, c, d, e, f) and reported in bold, in the Table 1.

These results, divided for LAM and SUF station, are summarized below.

\subsection{LAM Station}

Continuous FDDA (T36_W36) shows a mean RMSE reduction of $41 \%$ (a) for the complete run, and $63 \%$ (b) for the first 6 hours of simulation. The result (a) can be considered as representative of the improvement in the production of mesoscale analysis.

Forecasting configuration FDDA (T12_W12) shows a mean RMSE reduction of $16 \%$ (c) for the whole run, and $35 \%$ (d) for the first 6 hours. The result (d) can be considered as representative of the improvement in shortterm prediction. 
Table 1. Summary of temperature $\operatorname{MAE}\left({ }^{\circ} \mathrm{C}\right)$ and $\operatorname{RMSE}\left({ }^{\circ} \mathrm{C}\right)$ results (see text for explanation).

Temperature - MAE ( $\left.{ }^{\circ} \mathrm{C}\right)$

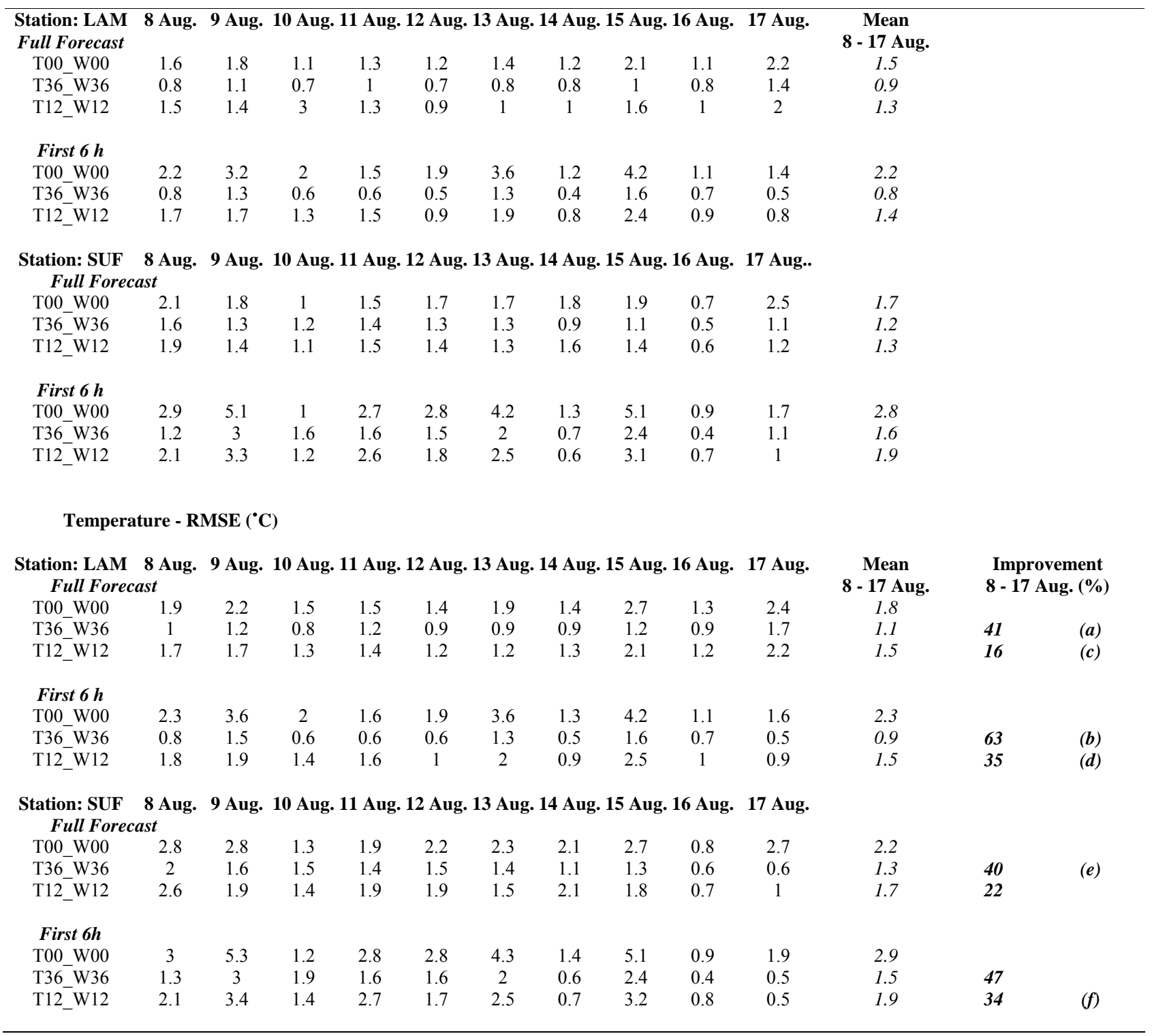

\subsection{SUF Station (Independent Verification)}

In this case, continuous FDDA (T36_W36) shows a RMSE reduction of $40 \%$ (e), on average, for the whole duration of run. The result (e), likewise to the (a), can be considered as representative of the model improvement in the production of mesoscale analysis at an "independent site".

Forecasting configuration FDDA (T12_W12) shows a mean RMSE reduction of 34\% (f) in the first 6 hours of run. The result (f), likewise to the (d), can be considered as the impact of the FDDA in the improvement of short- term prediction at an "independent site".

To better understanding the mechanism and the effects of data assimilation, two examples for the SUF station, related to two opposite situations, are discussed below.

The first case refers to the August 15 and shows good results. Figure 3 shows the daily temperature, measured (dotted line) and simulated, both before (dashed line) and after (solid line) the application of the assimilation procedure; more precisely, the T00_W00 and T36_W36 simulations are reported. From the figure, it follows the high improvement when assimilation procedure is applied. In particular, there is an error reduction of $53 \%$ in 


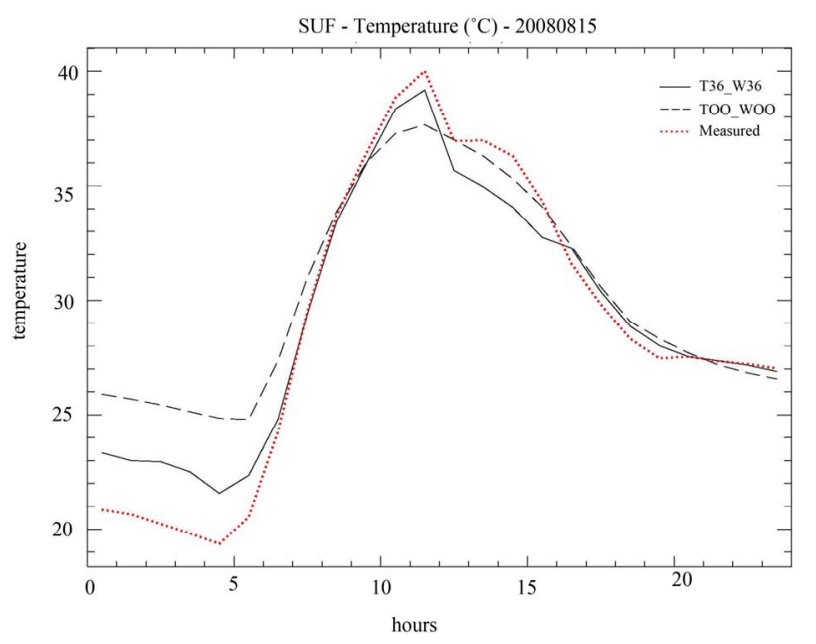

Figure 3. Simulated temperature (solid line for T36_W36, dashed line for T00_W00) and measured temperature (dotted line) for the $15 / 08 / 2008$.

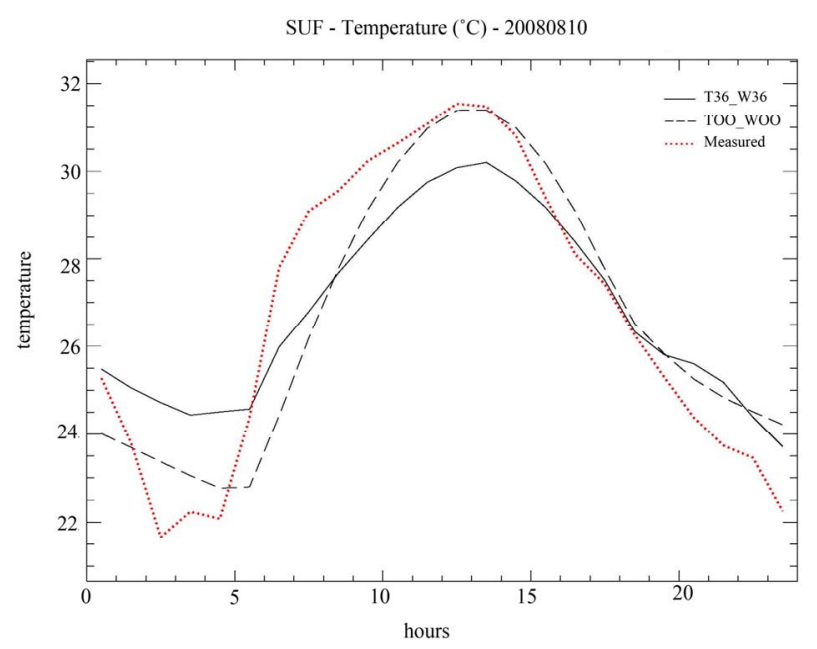

Figure 4. Simulated temperature (solid line for T36_W36, dashed line for T00_W00) and measured temperature (dotted line) for the $10 / 08 / 2008$.

the first six hours of run and $45 \%$ for the whole duration of the simulation. The assimilation procedure is strong and more effective in the first hours, when the difference between measured and simulated temperature is larger. This case, particularly favourable, shows the possibility to halving the model errors.

The second case refers to the August 10 and has poor results. We remark that for this day, the forecast error was low without data assimilation. In this particular case, moreover, the two considered stations show discrepancy in wind direction. Likely, the nocturnal land breeze has occurred in SUF but not in LAM, while the model simulated nocturnal breeze in both sites. For this particular case, the assimilation of LAM data worsened the simulation at SUF station instead to improving it. Figure 4 shows the statements just made, before and after the assimilation (dashed line for T00_W00, solid line for T36_W36 and dotted line for measurements). In this case, there is an error increment of $59 \%$ in the first six hours of run and $18 \%$ for the whole duration of the simulation.

As stated, this is a preliminary work of the FDDA evaluation performances. Our future interest, already subjected to study, is to realize a system that discriminates possible differences between distinct-but-close measuring stations, in order to avoid potential negative effects of the assimilation. Moreover, further studies will be devoted to test different nudging configurations. In particular, different values for the nudging weight and of the radius of nudging will be investigated.

\section{Conclusions}

A tailored version of the Regional Atmospheric Modeling System (RAMS) was preliminarily tested to investigate the improvements of the model performance, by the implementation of a four-dimensional data assimilation (FDDA) scheme based on a Newtonian relaxation ("nudging"). To cope with this issue, meteorological data available in the Central Mediterranean peninsula of Calabria, in southern Italy, were considered both for assimilation and for verification purposes. All data are derived from instruments operating routinely at the CRATI/ ISAC-CNR experimental field, located $600 \mathrm{~m}$ from the Tyrrhenian coastline.

We present results from the analyses of a ten-day case study, in summer 2008, where RAMS outputs at high spatial horizontal resolution $(1 \mathrm{~km})$ are considered.

For the assimilation, we combined observation from a wind profiler, a sodar, and a surface meteorological station. In particular, vertical wind profiles are derived by sodar and wind profiler. Instead, wind, temperature and specific humidity, are derived from the surface station (also used, subsequently, for verification). A second station, not far from the experimental field, is considered for independent verifications.

The model validation is performed for the surface temperature. The RAMS fields, simulated with and without data assimilation, were evaluated and compared for selected case studies, and several experiments were carried out for each case.

Results show that assimilating wind and temperature, throughout all the simulation time (continuous FDDA) and for a $12 \mathrm{~h}$ time window (forecasting configuration), produces a general improvement of the model performances.

At LAM, the mean improvement is considerable $(40 \%$ error reduction) in the case of continuous FDDA, while it is reduced in case of forecasting configuration $(15 \%$ to 
$35 \%$ error reduction, depending on cases). The improvements during the first 6 hours of run are generally higher (up to $60 \%$ for continuous FDDA).

An important outcome is that the independent verification provides good results. At SUF station, in fact, the improvement is of about $40 \%$ for continuous FDDA and, in the first 6 hours, the error reduction reaches $47 \%$.

The obtained meteorological fields are finalised for the initialization of air quality and agro-meteorological models, and also for the initial and boundary conditions of very high-resolution atmospheric models. At the same time, they may be very useful both for short-term meteorological forecast and for the production of gridded meteorological analysis.

Further analyses, however, are needed, to better understand the impact of the assimilation procedure in different coastal meteorological conditions.

\section{Acknowledgements}

This work was partially funded by "Regione Calabria" within the project "MAPVIC".

\section{References}

[1] E. Kalnay, "Atmospheric Modeling, Data Assimilation, and Predictability," Cambridge University Press, New York, 2002.

[2] R. A. Anthes, "Data Assimilation and Initialization of Hurricane Prediction Models," Journal of Atmospheric Science, Vol. 31, No. 3, 1974, pp. 702-719. doi:10.1175/1520-0469(1974)031<0702:DAAIOH >2.0.C $\underline{\mathrm{O} ; 2}$

[3] R. E. Kistler, "A Study of Data Assimilation Techniques in an Autobarotropic Primitive Equation Channel Model," Ph.D Dissertation, The Pennsylvania State University, University Park, 1974.

[4] D. R. Stauffer and N. L. Seaman, "Use of Four-Dimensional Data Assimilation in a Limited-Area Mesoscale Model. Part I: Experiments with Synoptic-Scale Data," Monthly Weather Review, Vol. 118, No. 6, 1990, pp. $1250-1277$. doi:10.1175/1520-0493(1990)118<1250:UOFDDA $>2.0$. $\mathrm{CO} ; 2$

[5] D. R. Stauffer and N. L. Seaman, "Multiscale Four-DiMensional Data Assimilation," Journal of Applied Meteorology, Vol. 33, No. 3, 1994, pp. 416-434. doi:10.1175/1520-0450(1994)033<0416:MFDDA >2.0.C $\underline{\mathrm{O} ; 2}$

[6] N. L. Seaman, D. R. Stauffer, G. K. Hunter and A. M. Lario-Gibbs, "The Use of the San Jaoquin Valley Meteorological Model in Preparation of a Field Program in the South Coast Air Basin and Surrounding Regions of Southern California. Vol. II." Numerical Modelling Studies in Support of Design for the 1997 Southern California Ozone Study (SCOS-97) Field program, Final Report,
California Air Resources Board, Sacramento, 1997.

[7] J. D. Fast, "Mesoscale Modeling and Four-Dimensional Data Assimilation in Areas of Highly Complex Terrain," Journal of Applied Meteorology, Vol. 34, No. 12, 1995, pp. 2762-2782.

doi:10.1175/1520-0450(1995)034<2762:MMAFDD $>2.0$. $\mathrm{CO} ; 2$

[8] N. L. Seaman, "Meteorological Modeling for Air Quality Assessments", Atmospheric Environment, Vol. 34, No. 12-14, 2000, pp. 2231-2259. doi:10.1016/S1352-2310(99)00466-5

[9] S. Tanrikulu, D. R. Stauffer, N. L. Seaman and A. J. Ranzieri, "A Field-Coherence Technique for Meteorological Field-Program Design for Air Quality Studies. Part II: Evaluation in the San Joaquin Valley," Journal of Applied Meteorology, Vol. 39, 2000, pp. 317-334. doi: 10.1175/1520-0450(2000)039<0317:AFCTFM $>2.0 . C$ $\mathrm{O} ; 2$

[10] S. A. Michelson and N. L. Seaman, "Assimilation of NEXRAD-VAD Winds in Summertime Meteorological Simulations over the North-Eastern United States," Journal of Applied Meteorology, Vol. 39, No. 3, 2000, pp. 367-383.

doi:10.1175/1520-0450(2000)039<0367:AONVWI $>2.0$. $\mathrm{CO} ; 2$

[11] J. W. Nielsen-Gammon, R. T. McNider, W. M. Angevine, A. B. White and K. Knupp, "Mesoscale Model Performance with Assimilation of Wind Profiler Data: Sensitivity to Assimilation Parameters and Network Configuration," Journal of Geophysical Research, Vol. 112, No. 9, 2007. doi:10.1029/2006JD007633

[12] T. Umeda and P. T. Martien, "Evaluation of a Data Assimilation Technique for a Mesoscale Meteorological Model Used for Air Quality Modelling," Journal of Applied Meteorology, Vol. 41, No. 1, 2002, pp. 12-29. doi:10.1175/1520-0450(2002)041<0012:EOADAT $>2.0$. $\mathrm{CO} ; 2$

[13] M. Barna and B. Lamb, "Improving Ozone Modeling in Regions of Complex Terrain Using Observational Nudging in a Prognostic Meteorological Model," Atmospheric Environment, Vol. 34, No. 28, 2000, pp. 4889-4906. doi:10.1016/S1352-2310(00)00231-4

[14] S. Federico, L. Pasqualoni, L. De Leo, C. Bellecci, "A Study of the Breeze Circulation during Summer and Fall 2008 in Calabria, Italy," Atmospheric Research, Vol. 97, No. 1-2, 2010, pp. 1-13. doi:10.1016/j.atmosres.2010.02.009

[15] S. Federico, L. Pasqualoni, A.M. Sempreviva, L. De Leo, E. Avolio, C.R. Calidonna and C. Bellecci, "The seasonal characteristics of the Breeze Circulation at a Coastal Mediterranean Site in South Italy," Advances in Science and Research, Vol. 4, 2010, pp. 47-56. doi:10.5194/asr-4-47-2010

[16] E. Avolio, L. Pasqualoni, S. Federico, M. Fornaciari, T. Bonofiglio, F. Orlandi, C. Bellecci and B. Romano, "Correlation Between Large Scale Atmospheric Fields and Olive Pollen Season in Central Italy," International Journal of Biometeorology, Vol. 52, No. 8, 2008, pp. 787-796. doi:10.1007/s00484-008-0172-5 
[17] S. Federico, E. Avolio, C. Bellecci and M. Colacino, "The Meteorological Model RAMS at Crati Scrl," Advances in Geosciences, Vol. 2, 2005, pp. 177-180. doi:10.5194/adgeo-2-177-2005

[18] W. R. Cotton, R. A. Pielke Sr., R. L. Walko, G. E. Liston, C. J. Tremback, H. Jiang, R. L. McAnelly, J. Y. Harrington, M. E. Nicholls, G. G. Carrio, J. P. McFadden, "RAMS 2001: Current Status and Future Directions," Meteorology and Atmosphere Physics, Vol. 82, No. 1-4, 2003, pp. 5-29. doi:10.1007/s00703-001-0584-9

[19] R. L. Walko, W. R. Cotton, M. P. Meyers, J. Y. Harrington, "New RAMS Cloud Microphysics Parameterization Part I: The Single-Moment Scheme," Atmosphere Research, Vol. 38, No. 1-4, 2995, pp. 29-62. doi:10.1016/0169-8095(94)00087-T

[20] J. Molinari and T. Corsetti, "Incorporation of Cloud-Scale and Mesoscale Down-Drafts Into A Cumulus Parameterization: Results of One and Three-Dimensional Integrations," Monthly Weather Review, Vol. 113, No. 4, 1985, pp. 485-501.
doi:10.1175/1520-0493(1985)113<0485:IOCSAM >2.0.C $\underline{\mathrm{O} ; 2}$

[21] R. L. Walko, L. E. Band, J. Baron, T. G. Kittel, R. Lammers, T. J. Lee, D. Ojima, R. A. Sr. Pielke, C. Taylor, C. Tague, C. J. Tremback and P. L. Vidale, "Coupled Atmosphere-Biosphere-Hydrology Models for Environmental Prediction," Journal of Applied Meteorology, Vol. 39, No. 6, 2000, pp. 931-944. doi:10.1175/1520-0450(2000)039<0931:CABHMF $>2.0$. $\mathrm{CO} ; 2$

[22] G. Mellor and T. Yamada, "Development of a Turbulence Closure Model for Geophysical Fluid Problems," Reviews of Geophysics and Space Physics, Vol. 20, No. 4, 1982, pp. 851-875. doi:10.1029/RG020i004p00851

[23] J. E. Hoke and R. A. Anthes, "Initialization of numerical Models by a Dynamic-Initialization Technique," Monthly Weather Review, Vol. 104, No. 12, 1976, pp. 1551-1556. doi:10.1175/1520-0493(1976)104<1551:TIONMB $>2.0 . \mathrm{C}$ $\underline{\mathrm{O} ; 2}$ 